\title{
SN 2010jp (PTF10aaxi): A Jet-driven Type II Supernova
}

Nathan Smith ${ }^{1} \dagger$, S. Bradley Cenko ${ }^{2}$, Nat Butler ${ }^{2}$, Joshua S. Bloom ${ }^{2}$, Mansi M. Kasliwal ${ }^{3}$, Assaf Horesh ${ }^{3}$, Shrinivas R. Kulkarni ${ }^{3}$, Nicholas M. Law ${ }^{4}$, Peter E. Nugent ${ }^{2,5}$, Eran O. Ofek ${ }^{3}$, Dovi Poznanski ${ }^{2,5}$, Robert M. Quimby ${ }^{3}$, Branimir Sesar ${ }^{3}$, Sagi Ben-Ami ${ }^{6}$, Iair Arcavi ${ }^{6}$, Avishay Gal-Yam ${ }^{6}$, David Polishook ${ }^{6}$, Dong Xu ${ }^{6}$, Ofer Yaron ${ }^{6}$, Dale A. Frail ${ }^{7}$, \& Mark Sullivan ${ }^{8}$

${ }^{1}$ Steward Observatory, University of Arizona, 933 North Cherry Ave., Tucson, AZ 85721, USA

${ }^{2}$ Department of Astronomy, University of California, Berkeley, CA 94720-3411, USA

${ }^{3}$ Cahill Center for Astrophysics, California Institute of Technology, Pasadena, CA, 91125, USA

${ }^{4}$ Dunlap Institute for Astronomy and Astrophysics, University of Toronto, 50 St. George Street, Toronto M5S 3H4, Ontario, Canada

${ }^{5}$ Computational Cosmology Center, Lawrence Berkeley National Laboratory, 1 Cyclotron Road, Berkeley, CA 94720, USA

${ }^{6}$ The Weizmann Institute of Science, Rehovot 76100, Israel

${ }^{7}$ National Radio Astronomy Observatory, P.O. Box O, Socorro, NM 87801, USA

${ }^{8}$ Department of Physics, University of Oxford, Keble Road, Oxford, OX13RH, UK

\begin{abstract}
We present photometry and spectroscopy of the peculiar Type II supernova SN 2010jp, also named PTF10aaxi. The light curve exhibits a linear decline with a relatively low peak absolute magnitude of only -15.9 (unfiltered), and a low radioactive decay luminosity at late times that suggests a low synthesized nickel mass of about $0.003 M_{\odot}$ or less. Spectra of SN 2010jp display an unprecedented triple-peaked $\mathrm{H} \alpha$ line profile, showing: (1) a narrow central component that suggests shock interaction with a dense circumstellar medium (CSM); (2) high-velocity blue and red emission features centered at $-12,600$ and $+15,400 \mathrm{~km} \mathrm{~s}^{-1}$; and (3) very broad wings extending from $-22,000$ to $+25,000 \mathrm{~km} \mathrm{~s}^{-1}$. We propose that this line profile indicates a bipolar jet-driven explosion, with the central component produced by normal SN ejecta and CSM interaction at mid and low latitudes, while the high-velocity bumps and broad line wings arise in a nonrelativistic bipolar jet. Jet-driven SNe II are predicted for collapsars resulting from a wide range of initial masses above $25 M_{\odot}$, especially at the sub-solar metallicity consistent with the SN host environment. It also seems consistent with the apparently low ${ }^{56} \mathrm{Ni}$ mass that may accompany black hole formation. We speculate that the jet survives to produce observable signatures because the star's H envelope was very low mass, having been mostly stripped away by the previous eruptive mass loss.
\end{abstract}

Keywords. ISM: jets and outflows, supernovae: general

\section{Introduction}

Many theoretical studies of core-collapse suggest that breaking spherical symmetry may be an essential ingredient for producing a successful SN explosion (Blondin et al. 2003; Buras et al. 2006a, 2006b; Burrows et al. 2006, 2007). An extreme case of breaking spherical symmetry involves jet-driven explosions (Khokhlov et al. 1999; Höflich et al. 2001; Maeda \& Nomoto 2003; Wheeler et al. 2000; Couch et al. 2009). Strongly collimated

$\dagger$ Email: nathans@as.arizona.edu 
jets that expel the surrounding stellar envelopes may arise from accretion onto newlyformed black holes as in the "collapsar" model (MacFadyen \& Woosley 1999; MacFadyen et al. 2001), or by magnetohydrodynamic (MHD) mechanisms in the collapse and spindown of highly magnetized and rapidly rotating neutron stars, or magnetars (LeBlanc \& Wilson 1970; Bodenheimer \& Ostriker 1974; Wheeler et al. 2000; Thompson et al. 2004; Bucciantini et al. 2006; Burrows et al. 2007; Komissarov \& Barkov 2007; Dessart et al. 2008; Metzger et al. 2010; Piro \& Ott 2011). A jet-driven explosion may be important for producing successful SNe from high-mass stars above $\sim 25 M_{\odot}$ (e.g., MacFadyen et al. 2001; Heger et al. 2003), which might otherwise collapse quietly to a black hole.

While there is strong evidence of collimated jets in broad-lined SNe Ic from the relativistic jets that produce observable gamma-ray bursts (GRBs) (Woosley \& Bloom 2006; Galama et al. 1998; Matheson et al. 2003; Mazzali et al. 2005; see also Soderberg et al. 2009), there is to-date no substantial evidence for collimated jets in Type II supernovae. Jets are expected to not survive passage through the larger and more massive $\mathrm{H}$ envelope of a typical RSG (MacFadyen et al. 2001; Höflich et al. 2001), and this may help account for some of the time-dependent asymmetry inferred from polarization studies of SNe II-P (Leonard et al. 2001, 2006).

In this paper we discuss the recent peculiar Type II SN 2010jp (also called PTF10aaxi), which shows evidence for a fast (although non-relativistic) collimated jet that survived passage through its $\mathrm{H}$ envelope, perhaps because of significant previous mass loss. This

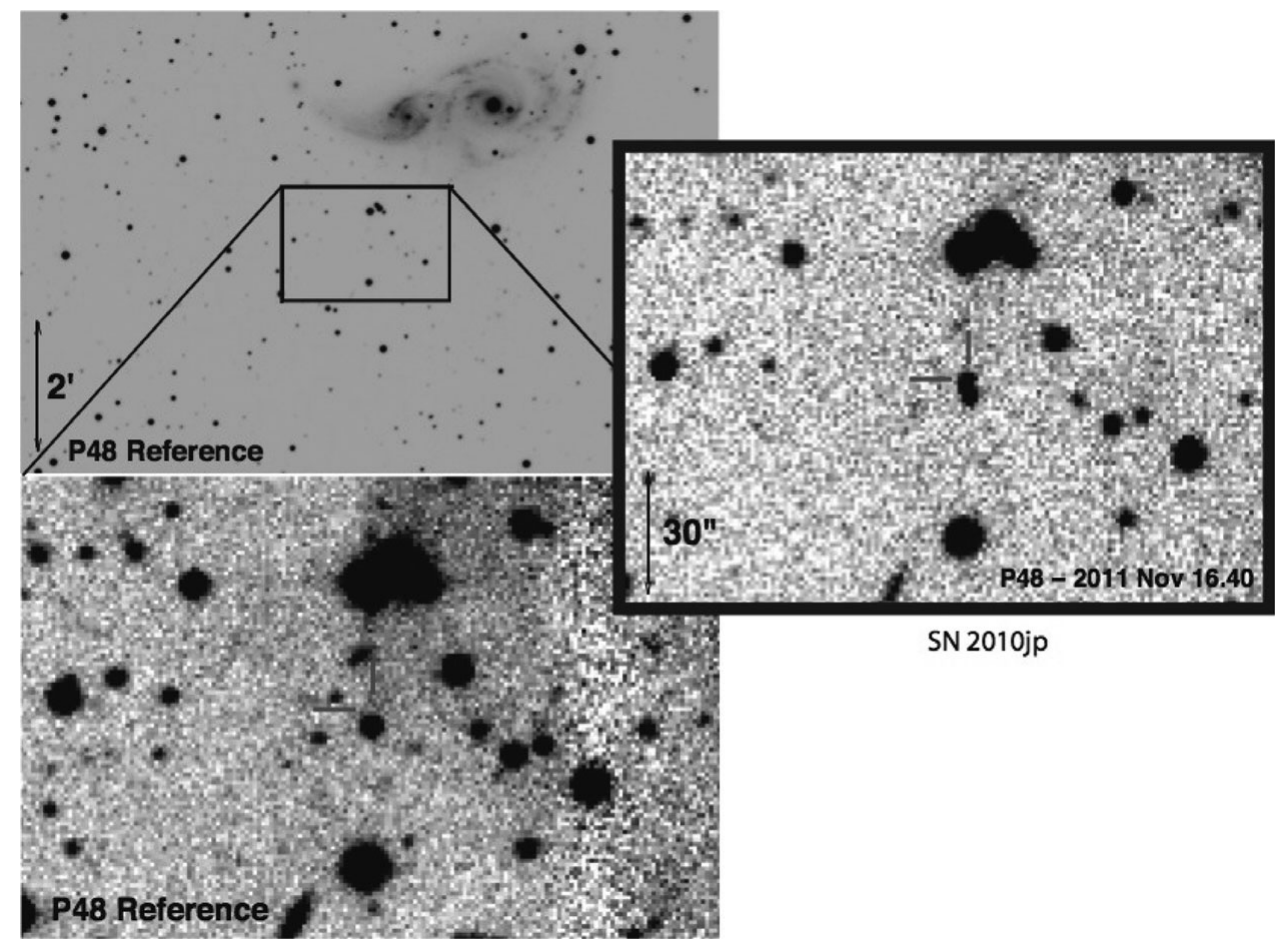

Figure 1. Finder chart for SN 2010jp (PTF10aaxi). Top Left: Wide-field P48 image of the SN field, including pre-discovery frames taken from 2009 November to $2010 \mathrm{March}$. The interacting galaxy pair IC 2163 / NGC 2207 is clearly visible about 2 arcminutes to the north. Bottom Left: Zoomed-in portion of the same P48 reference image of the location of SN 2010jp. Right: P48 image of SN2010jp. The SN position, indicated with the red tick marks, is a few arcseconds north of a foreground star, which appears blended with the P48 angular resolution. 
talk gives only an overview of work that is now published in Smith et al. (2012); more detail about the analysis and additional references can be found there.

\section{Observation Summary}

Imaging/Host Galaxy. Images of the site of SN 2010jp obtained with the Palomar 48 -inch (P48) telescope ( $R$-band) are shown in Figure 1 . This provides our first important clue about the nature of SN 2010jp: it occurs in a remote environment. There is nothing visible at the position of SN 2010jp in pre-explosion images, and upper limits there suggest a very faint dwarf galaxy at least 40 times fainter than the SMC. Alternatively, SN 2010jp may be in the far outer regions of the pair of interacting galaxies NGC 2207/IC 2163 (top panel of Fig. 1). NGC 2207 has a redshift of $z=0.0091$, which is the same as SN 2010jp, so if SN 2010jp is associated with these host galaxies, it is located $33 \mathrm{kpc}$ out from their center. In either case, SN 2010jp probably occurred in a relatively low-metallicity environment that is comparable to the SMC or even more metal-poor.

Photometry. We analyzed photometry obtained with the Palomar 48-inch (P48) telescope ( $R$-band), the Palomar 60-inch (P60) telescope ( $g, r$, and $i$ bands), and UV/optical photometry obtained with Swift (uvw2, uvm2, uvw1, $u, b$, and $v$ bands). The P48 $R$-band light curve is shown in Fig. 2 (additional photometry is presented in Smith et al. 2012). Basically, the light curve of SN 2010jp looks like an underluminous light curve of a Type II-L or IIn supernova. Its peak absolute magnitude is about -15.9 , reached about 20 days after discovery, from which it fades slowly until 110 days after discovery. After that point, it appears to fade faster (Fig. 2), although this late phase fading or a possible radioactive decay tail are not well constrained. Figure 2 shows a representative upper limit to the radioactive decay luminosity, corresponding to a ${ }^{56} \mathrm{Ni}$ mass of only 0.003 $M_{\odot}$. This indicates that SN 2010jp seems to have produced very little ${ }^{56} \mathrm{Ni}$ compared to normal core-collapse SNe.

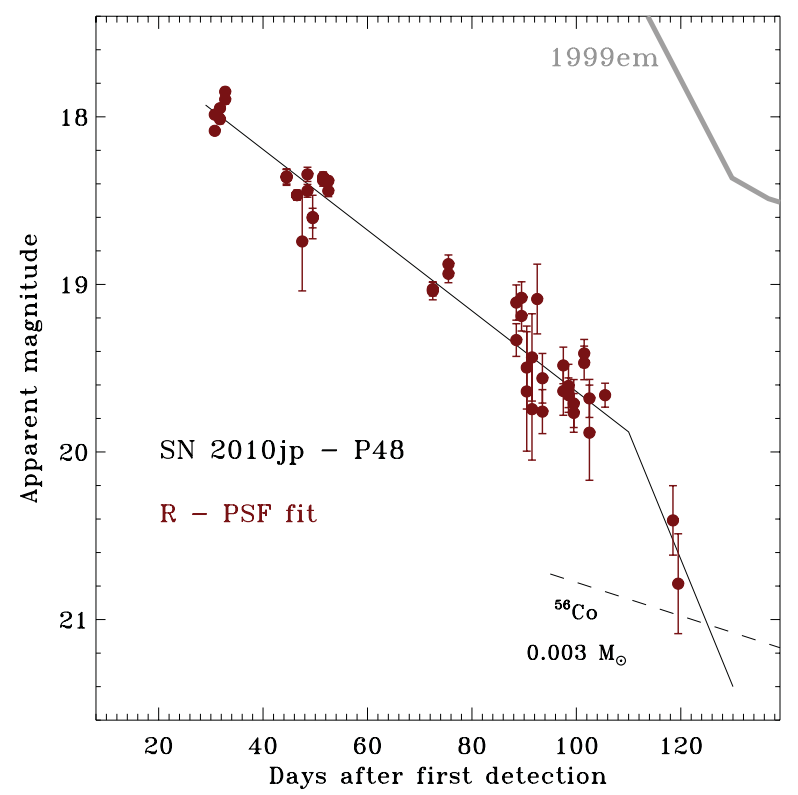

Figure 2. Light curve of SN 2010jp, plotted as days after the first detection. We show $R$-band magnitudes from the Palomar 48-in telescope, reduced using PSF-fitting photometry (see Smith et al. 2012). 
Spectroscopy. We obtained a series of visual-wavelength spectra with the $M M T$ (bluechannel spectrograph), the Palomar 5m (Double-beam spectrograph), and the Keck telescope (LRIS spectrograph). Our series of spectra are shown in Figure 3. The most striking aspect of the spectra in Figure 3 is the strong $\mathrm{H} \alpha$ line with an extremely unusual triplepeaked line profile, simultaneously showing extremely broad wings $\left( \pm 25,000 \mathrm{~km} \mathrm{~s}^{-1}\right)$ and a narrow core (few $10^{2} \mathrm{~km} \mathrm{~s}^{-1}$ ). A more detailed view of the $\mathrm{H} \alpha$ line is shown in Figure 4. This triple-peaked profile is the central aspect of the observations that point toward the presence of a collimated jet in SN 2010jp. Also noteworthy is the marked absence of any lines in the spectrum other than the H Balmer lines (i.e., no Ca II or Fe II lines that are usually seen in Type II SNe), which supports the conjecture that SN 2010jp arose in a low-metallicity environment.

Radio/X-ray. The site of SN 2010jp was observed at radio wavelengths with the EVLA and at X-rays using Swift. Details of the observations are presented in Smith et al. (2012). Both sets of observations yielded only non-detections at a significant level, which provide important arguments that the multiple peaks in the $\mathrm{H} \alpha$ profile in SN 2010jp were not associated with accretion onto a massive black hole.

\section{Interpretation}

More detail about the interpretation of SN 2010jp is given in Smith et al. (2012), but here we briefly list the essential components of the observations:

1. SN 2010jp occurred in a remote, low-metallicity environment.

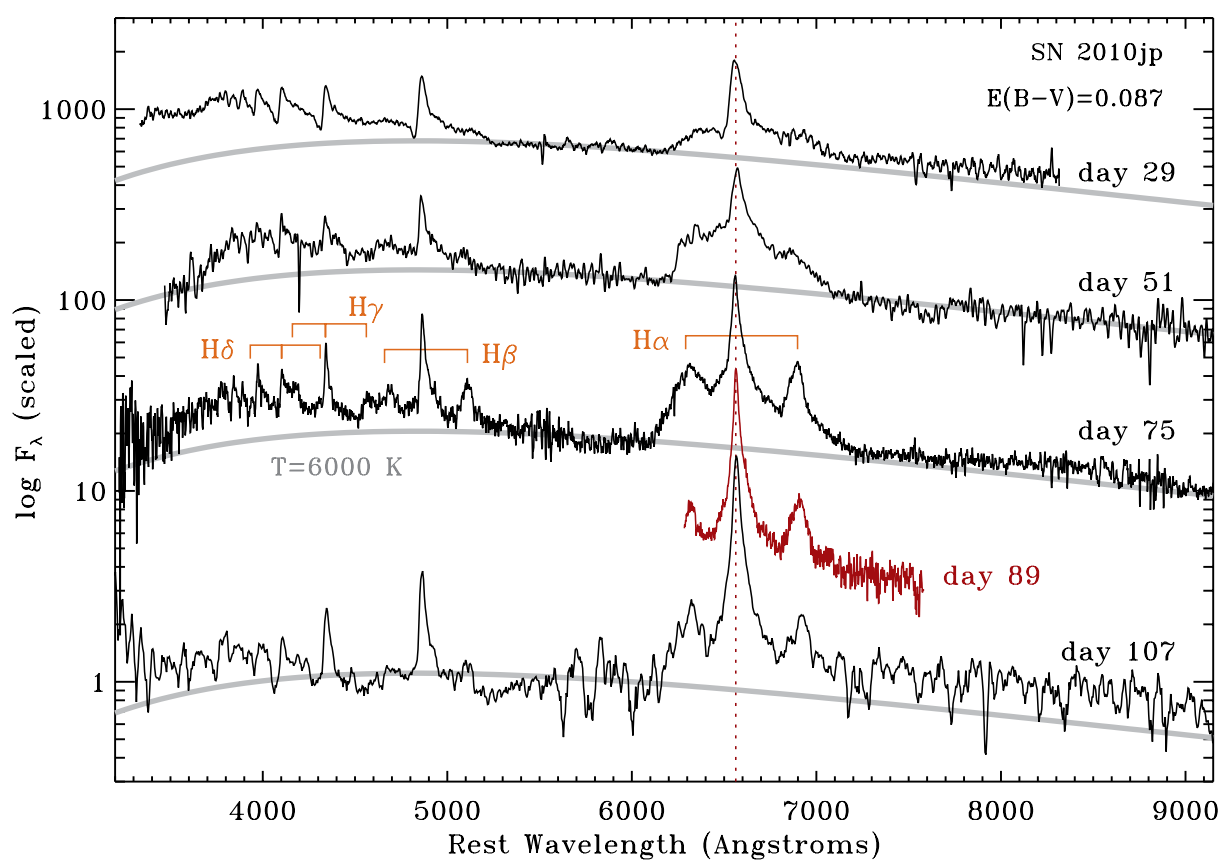

Figure 3. Visual-wavelength spectra of SN 2010jp obtained with the MMT, the Palomar 200-in telescope, and Keck, listed as days after discovery. The day 89 spectrum plotted in red is a high-resolution spectrum from the MMT. The blue and red high-velocity components of $\mathrm{H} \alpha$, $\mathrm{H} \beta, \mathrm{H} \gamma$, and $\mathrm{H} \delta$ are marked in orange brackets on the day 75 spectrum taken with Keck/LRIS. 
2. A strange triple-peaked $\mathrm{H} \alpha$ profile (the triple peak is also seen in other lines of the Balmer series). On either side of the central narrow peak (see below), we see distinct blue and redshifted peaks at roughly $\pm 15,000 \mathrm{~km} \mathrm{~s}^{-1}$.

3. The central narrow peak has a width of about $800 \mathrm{~km} \mathrm{~s}^{-1}$, resolved in our day 89 MMT spectrum. This probably corresponds to the speed of the CSM of the progenitor star, where the central part of the line profile arises from CSM interaction.

4. From the light curve, SN 2010jp was not particularly luminous for core-collapse SNe, with a peak absolute $R$ magnitude of about -15.9 mag. This is on the faint tail of the luminosity function for SNe II-L and IIn (Li et al. 2011), which tend to have similarly shaped light curves (see additional figures in Smith et al. 2012). CSM interaction therefore does not provide a substantial luminosity boost for SN 2010jp. The progenitor star's mass-loss rate was probably not more than about $10^{-3} M_{\odot} \mathrm{yr}^{-1}$ (Smith et al. 2009).

5. From the late time luminosity, we find that SN 2010jp had a ${ }^{56} \mathrm{Ni}$ mass less than $0.003 M_{\odot}$, which is $\sim 10$ times less than a prototypical SN II-P like SN $1999 \mathrm{em}$. This low ${ }^{56} \mathrm{Ni}$ mass could indicate either that the progenitor star had a relatively low initial mass near $8 M_{\odot}$, or that it was underluminous because it lost much of the radioactive material into a black hole.

6. The non-detection of radio or X-ray emission argues against an interpretation where the blue and red peaks in the $\mathrm{H} \alpha$ line arise from accretion around a massive black hole, as

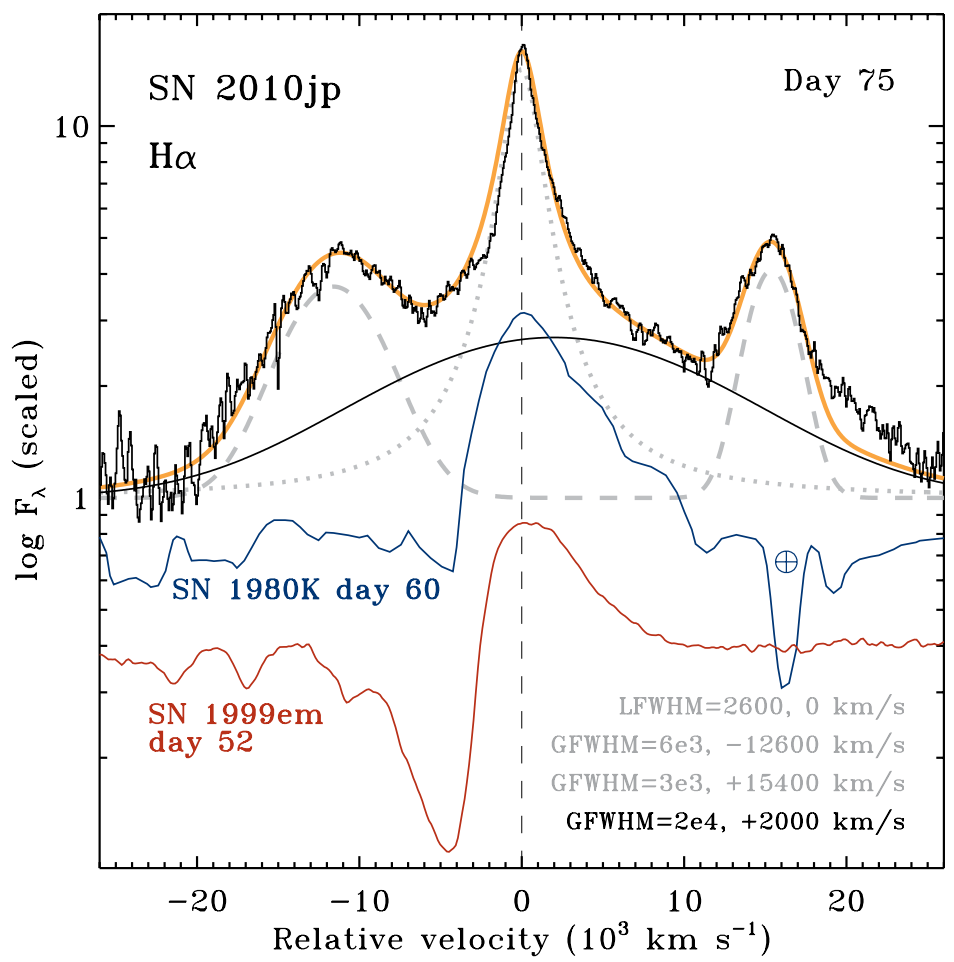

Figure 4. The H $\alpha$ profile of SN 2010jp on day 75, decomposed into multiple contributing features (thin black and grey curves) with the sum of all individual components shown in orange. GFWHM and LFWHM denote Gaussian or Lorentzian FWHM values and centroid velocities. For comparison, we also show the $\mathrm{H} \alpha$ profile of the normal SN II-P $1999 \mathrm{em}$ from Leonard et al. (2002), plotted in red, as well as the $\mathrm{H} \alpha$ line in the Type II-L SN 1980K observed on day $~ 60$ (Barbieri et al. 1982) in blue. 
in the class of double-peaked emitters among AGN (Halpern \& Filippenko 1988). There are other arguments against this interpretation based on the time evolution of velocity, the requirements on black hole mass, and the remote environment (see Smith et al. 2012).

We speculate that the observed properties of SN 2010jp arises from a superposition of two physical scenarios. The first is a relatively traditional Type IIn explosion, where the rapidly expanding low-mass $\mathrm{H}$ envelope of the star collides with dense pre-existing CSM ejected recently by the progenitor. This can produce the blue continuum, the narrow emission cores of the Balmer lines, and some of the underlying broad emission profiles (see Smith et al. 2008). Most of the emitting volume corresponds to this component (regions 1, 2, and 3 in Figure 5). The second component is that SN 2010jp also produces a fast bipolar jet, tilted out of the plane of the sky, which gives rise to the fast blue and red emission features in $\mathrm{H} \alpha$, and some emission in the very broad line wings. The combination of these two scenarios is depicted schematically in Figure 5. The two isolated red and blue emitting components must arise in a collimated geometry, as explained further in Smith et al. (2012).

The detection of a collimated jet in a Type II explosion is unprecedented. Models of jet-powered SNe have been published for fully stripped-envelope progenitors, which yield

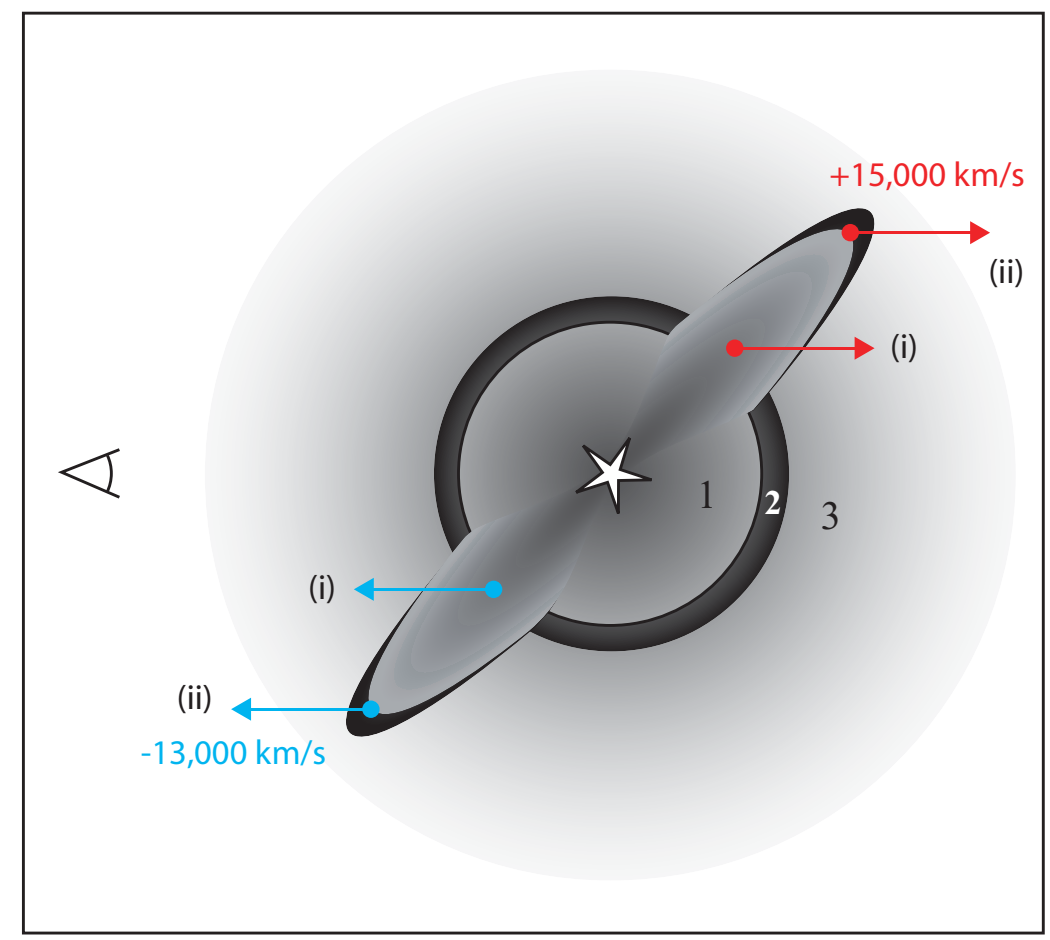

Figure 5. Cartoon of the possible jet-powered geometry in SN 2010jp. Regions 1, 2, and 3 correspond to the unshocked SN ejecta (inner gradient), the CSM interaction region (dark), and the pre-shock CSM (outer gradient), respectively. The upper-left and lower-right quadrants in this cartoon are the same as for a conventional SN IIn with CSM interaction, corresponding to low/mid-latitudes in the explosion. The lower-left and upper-right quadrants depict a tilted fast bipolar jet breaking through the otherwise spherical CSM interaction shell. An observer located to the left would see a combination of the spectrum from a conventional SN IIn, plus blue and red emission peaks in emission lines arising in either the unshocked jet material (i) or at the reverse shock in the jet (ii). We suggest that a bipolar jet such as this causes the blue and red bumps at $-13,000$ and $+15,000 \mathrm{~km} \mathrm{~s}^{-1}$ observed in the H $\alpha$ profile of SN 2010jp. 
SNe Ibc and GRBs. Theoretical models also predict jet-driven SNe II for a wide range of initial masses exceeding $25 M_{\odot}$ (e.g., Heger et al. 2003), from collapsars that yield black holes. These are expected to be more common at sub-solar metallicity (Heger et al. 2003), due to the expectation of weaker metallicity-dependent mass loss.

We consider two possible specific mechanisms that may power the emission in the fast blue and red peaks of the $\mathrm{H}$ Balmer series: (i) A jet-powered explosion might mix significant quantities of ${ }^{56} \mathrm{Ni}$ to high velocities in the polar regions of a thin $\mathrm{H}$ envelope. It is interesting that the $\mathrm{H} \alpha$ profile we observe bears a striking resemblance to the distribution of ${ }^{56} \mathrm{Ni}$ velocities in simulations of jet-powered SNe (Couch et al. 2009; see their Figure 12). (ii) Alternatively, the fast blue and red $\mathrm{H} \alpha$ bumps may be excited by CSM interaction. Fast H-bearing SN ejecta that cross the reverse shock of the jet will also yield two very fast but localized velocity components (Figure 5). Further study of additional examples of similar types of SNe, possibly including spectropolarimetry, might help solve this ambiguity.

\section{References}

Blondin, J. J. M., Mezzacappa, A., \& DeMarino, C. 2003, ApJ, 584, 971

Bodenheimer, P. \& Ostriker, J. P. 1974, ApJ, 191, 465

Bucciantini, N., Thompson, T. A., Aarons, J., Quataert, E., \& Del Zanna, L. 2006, MNRAS, 368,1717

Buras, R., Rampp, M., Janka, H. T., \& Kifonidis, K. 2006a, A\& A, 447, 1049

Buras, R., Janka, H. T., Rampp, M., \& Kifonidis, K. 2006b, A\& A, 457, 281

Burrows, A., Livne, E., Dessart, L., Ott, C. D., \& Murphy, J. 2006, ApJ, 640, 878

Burrows, A., Dessart, L., Livne, E., Ott, C. D., \& Murphy, J. 2007, ApJ, 664, 416

Couch, S. M., Wheeler, J. C., \& Milosavljević, M. 2009, ApJ, 696, 953

Dessart, L., Burrows, A., Livne, E., \& Ott, C. D. 2008, ApJ, 673, L43

Galama, T. J., et al. 1998, Nature, 395, 670

Halpern, J. P. \& Filippenko, A. V. 1988, Nature, 331, 46

Heger, A., Fryer, C. L., Woosley, S. E., Langer, N., \& Hartmann, D. H. 2003, ApJ, 591, 288

Höflich, P. A., Kholkov, A., \& Wang, L. 2001, in 20th Texas Symp. on Relativistic Astroph., eds. J.C. Wheeler, \& H. Martel (New York: AIP), 459

Khokhlov, .M., Höflich, P. A., Oran, E. S., Wheeler, J. C., Wang, L., \& Chtchelkanova, A. Y. 1999, ApJ, 524, L107

Komissarov, S. S. \& Barkov, M. V. 2007, MNRAS, 382, 1029

LeBlanc, J. M. \& Wilson, J. R. 1970, ApJ 161, 541

Leonard, D. C., Filippenko, A. V., Ardila, D. R., \& Brotherton, M. S. 2001, ApJ, 553, 861

Leonard, D. C., et al. 2006, Nature, 440, 505

Li, W., et al. 2011, MNRAS, 412, 1441

MacFadyen, A. I. \& Woosley, S. E. 1999, ApJ, 524, 262

MacFadyen, A. I., Woosley, S. E., \& Heger, A. 2001, ApJ, 550, 410

Maeda, K. \& Nomoto, K. 2003, ApJ, 598, 1163

Matheson, T., et al. 2003, ApJ, 599, 394

Mazzali, P. A., et al. 2005, Science, 308, 1284

Metzger, B. D., Giannios, D., Thompson, T. A., Bucciantini, N., \& Quataert, E. 2010, arXiv:1012.0001

Piro, A. L. \& Ott, C. D. 2011, ApJ, 736, 108

Smith, N., Chornock, R., Li, W., Ganeshalingam, M., Silverman, J. S., Foley, R., Filippenko, A. V., \& Barth, A. J. 2008a, ApJ, 686, 467

Smith, N., Hinkle, K. H., \& Ryde, N. 2009, AJ, 137, 3558

Smith, N., et al. 2012, MNRAS, 420, 1135

Soderberg, A., et al. 2009, Nature, 463, 513

Thompson, T. A., Chang, P., \& Quataert, E. 2004, ApJ, 611, 380 
Wheeler, J. C., Yi, I., Höflich, P., \& Wang, L. 2000, ApJ, 537, 810

Woosley, S. E. \& Bloom, J. S. 2006, ARAA, 44, 507

\section{Discussion}

KunCARAYAKTi: Are there any plans to study the host galaxy in more detail? (If it is an invisible dwarf galaxy.)

Smith: Yes, we would like to do this, but it will be difficult and will require HST due to the relatively bright field star located only an arcsecond away from the SN position.

Crowther: What is a massive star doing so far from its likely host? Does the redshift match that of the spiral galaxy pair?

Smith: Good question. Runaway? Tidal stream? eh. Anyway, the redshifts do match.

Folatelli: Have you considered a merger for your supernova SN 2010jp? That could explain the distance to any star forming region.

SмIтн: Sure, a late merger may also be a possibility, giving angular momentum, etc., but we don't have any observational constraints that can test this hypothesis. Strong asymmetry in the CSM might be an interesting avenue to pursue, though. [Author's note: There was an interesting paper by $\mathrm{R}$. Chevalier posted to the arXiv after this meeting, which is relevant here.]

MirABEL: Comment: The massive progenitor of SN 2010jp may have been formed in the low metallicity debris of the colliding galaxies that form faint "tidal dwarf galaxies".

SмIтH: I suppose this is possible; the red images of the SN 2010jp environment show some faint diffuse emission connecting back to the pair of interacting galaxies.

MAEDA: Do you have an idea about the rates of these kinds of objects? If you look at the phases earlier than the late phase with the triple-peaked H-alpha, are there any SNe IIn/II-L similar to this one.

Sмiтh: We have not seen anything similar to this before, so they are rare. I'm hesitant to quote a rate based on one relatively low-luminosity object in a rare/remote environment not sampled by the KAIT survey, but I'd guess substantially less than $1 \%$ of all core collapse SNe.

MAZZALI: Regarding a comment made by another audience member in the discussion (which was not recorded above) that the $\mathrm{H} \alpha$ profile could be produced by motion of the SN ejecta through an inhomogeneous shell: this comment does not apply here; if screening by a shell were the source of the $\mathrm{H} \alpha$ profile, the ejecta would need to be moving at $50,000 \mathrm{~km} \mathrm{~s}^{-1}$ or more. 